\title{
MicroRNA-125a-5p targets LIM kinase 1 to inhibit cisplatin resistance of cervical cancer cells
}

\author{
YONGQIAN XU* ${ }^{*}$ YUJIE ZHENG ${ }^{*}$, YAN DUAN, LIN MA and PING NAN \\ Department of Obstetrics and Gynecology, Shengli Oilfield Central Hospital, Dongying, Shandong 257000, P.R. China
}

Received January 11, 2020; Accepted January 28, 2021

DOI: 10.3892/ol.2021.12653

\begin{abstract}
Cervical cancer (CC), also known as invasive cervical carcinoma, is one of the most common gynecologic malignancies. The aim of the present study was to investigate the function of microRNA (miR)-125a-5p on CC progression and cisplatin (DDP) resistance. For this purpose, reverse transcription-quantitative PCR (RT-qPCR) was used to assess the expression of miR-125a-5p and LIMK1 in CC tissues, corresponding normal tissues and cells (human CC cell lines: C-33A, CaSKi; human cervical epithelial cells: HUCEC). Cisplatin (DDP) resistant cervical cancer cell lines were established (C-33A/DDP and CaSKi/DDP cell lines). RT-qPCR results demonstrated that miR-125a-5p or LIM kinase 1 (LIMK1) expression was downregulated or upregulated in C-33A/DDP and CaSKi/DDP cells, respectively. MTT assay, flow cytometry analysis and Western blotting were employed to detect the proliferation, apoptosis rate, $\mathrm{IC}_{50}$ of DDP and the expression of drug resistance-related proteins (P-glycoprotein and glutathione S-transferase- $\pi$ ). The targeting relationship between miR-125a-5p and LIMK1 was confirmed by the TargetScan database and dual-luciferase reporter gene assay. In CC tissues and cell lines, compared with normal tissues or HUCEC, miR-125a-5p expression was downregulated and LIMK1 expression was upregulated. The transfection with miR-125a-5p mimics decreased the proliferation of CaSKi/DDP cells, increased the apoptosis rate, reduced the $\mathrm{IC}_{50}$ of DDP, and downregulated the expression of drug resistance-related proteins; conversely, LIMK1 overexpression decreased the apoptosis rate, increased the $\mathrm{IC}_{50}$ of DDP, and upregulated the expression of drug resistance-related proteins. The luciferase reporter gene assay demonstrated that miR-125a-5p targeted and negatively regulated LIMK1.
\end{abstract}

Correspondence to: Dr Ping Nan, Department of Obstetrics and Gynecology, Shengli Oilfield Central Hospital, 31 Jinan Road, Dongying, Shandong 257000, P.R. China

E-mail: zhaoanzhong683@163.com

*Contributed equally

Key words: microRNA-125a-5p, cervical cancer, LIM kinase 1, cisplatin
miR-125a-5p could partially reverse the effect of LIMK1 on the proliferation, apoptosis, $\mathrm{IC}_{50}$ of DDP and the expressions of drug resistance-related proteins. The findings of the present study indicated that miR-125a-5p sensitizes CC cells to DDP by targeting LIMK1, hence increasing the anticancer efficacy of cisplatin.

\section{Introduction}

Cervical carcinoma (CC) is one of the most common tumors among women in the world, and the incidence of which was $\sim 7 \%$ in globally 2020 (1). In developed economies, the 5 -year survival rate of patients with $\mathrm{CC}$ is $>65 \%$, whereas this proportion is $<20 \%$ in developing countries (2). Chemotherapy is currently the main treatment for the patients suffering from advanced or recurrent CC (3). Cisplatin (DDP) is widely used in chemotherapy as it blocks DNA replication, inhibits cell cycle progression, induces apoptosis and hinders tumor growth (4). DDP has a prominent effect on CC treatments, DDP-based concurrent chemoradiotherapy is a standard treatment for locally advanced cervical cancer (3); irinotecan administered alone or in combination with DDP is useful in the treatment of recurrent cervical cancer (4). However, long-term use of DDP can lead to drug resistance in tumor cells, depriving patients of favorable therapeutic efficacy (5). Hence, methods that can lower the drug resistance of tumor cells are of great significance for improving the treatment of patients with $\mathrm{CC}$.

MicroRNA (miRNA), a class of conservative non-coding RNA, widely exists in eukaryotic cells and participates in biological processes, such as cell proliferation, differentiation and apoptosis, which are closely related to tumor progression and drug resistance of tumor cells (6-8). For instance, miR-296-5p enhances the drug resistance of pancreatic cancer cells leading to unfavorable prognosis in patients with pancreatic cancer (9). miR-21 is upregulated in DDP-resistant $\mathrm{CC}$ tissues and targets PTEN to promote drug resistance of CC (10). A study has demonstrated that miR-125a-5p reduces the resistance to imatinib in gastrointestinal stromal tumor (11). However, in CC cells, the function and mechanism of miR-125a-5p in regulating chemosensitivity remain unclear.

LIM kinase 1 (LIMK1) is a serine/threonine kinase belonging to the LIM kinase family that modulates actin polymerization through phosphorylation of the actin-binding factor cofilin 1 , which subsequently modulates cell motility and cell cycle (12). Studies have reported that LIMK1 
participates in the multidrug resistance of cancer $(13,14)$. For instance, LIMK1 promotes the migration and the invasion of non-small cell lung cancer (NSCLC) cells and facilitates the resistance of NSCLC cells to DDP (14). However, in CC, the influence of LIMK1 on the resistance of cancer cells to DDP and its mechanism warrants further research.

The present study aimed to provide further insight on the effect of miR-125a-5p and LIMK1 on CC cell viability and apoptosis and validate the interaction between miR-125a-5p and LIMK1. The findings of the present study may have important implications for treatment of cisplatin resistance of CC.

\section{Materials and methods}

Ethics and sample collection. From April 2017 to April 2018, a total of 45 patients who had been diagnosed with CC were enrolled and the surgically resected tumor tissues and corresponding adjacent tissues (at least $5 \mathrm{~cm}$ from the tumor) were collected form the Department of Pathology of Shengli Oilfield Central Hospital (Dongying, China). All the resected tissues were immediately placed in liquid nitrogen and stored in a cryogenic chamber at $-80^{\circ} \mathrm{C}$. All of the patients were diagnosed via biopsy and received radical hysterectomy for cervical cancer and did not receive any neoadjuvant radiation, neoadjuvant chemotherapy, or immunotherapy. The patients age range was 28-65 years (mean age, 46.5 years, median values, 44 years). The present study was given approval by the Medical Ethics Committee of Shengli Oilfield Central Hospital (Dongying, China) (approval no. 2016-05). All patients provided written informed consent prior to surgery.

Cell culture. Human CC cell lines (C-33A, CaSKi) and human cervical epithelial cells (HUCEC) were purchased from the China Infrastructure of Cell Line Resources, Institute of Basic Medical Sciences, Chinese Academy of Medical Sciences. The DDP resistant C-33A/DDP and CaSKi/DDP cell lines were established by treating C-33A and CaSKi cells with increasing DDP concentration. The concentration of cisplatin was increased gradually in the medium of C-33A and CaSKi cells from $0.10,0.50,1.00,1.25$ to $2.50 \mu \mathrm{g} / \mathrm{ml}$. After 2 weeks of culture, the cell morphology was observed. Subsequently, a high concentration of cisplatin, 2.505 .00 and $10.00 \mu \mathrm{g} / \mathrm{ml}$ was applied to C-33A and CaSKi cells. Following this method, C-33A and CaSKi cells were continuously cultured for 6 months. Finally, C-33A/DDP and CaSKi/DDP cells were cultured in medium supplemented with $10.00 \mu \mathrm{g} / \mathrm{ml}$ cisplatin to maintain the phenotype of cisplatin resistance. All of the cell lines mentioned above were maintained in Dulbecco's modified Eagle's medium (DMEM; HyClone; Cytiva) containing $10 \%$ fetal bovine serum (FBS; Hyclone, Cytiva) 100 U/1 penicillin and $100 \mathrm{mg} / \mathrm{l}$ streptomycin (both from Thermo Fisher Scientific Inc.) at $37^{\circ} \mathrm{C}$ in $5 \% \mathrm{CO}_{2}$. Medium was changed every 2 days and subculture was carried out when the cells reached $\sim 85 \%$ confluence.

Cell transfection. Over-expressing-LIMK1 plasmid (LIMK1-OE), empty plasmid (NC), siRNA targeting LIMK1 (si-LIMK1), siRNA negative control (si-NC), miRNA mimics normal control (miR-NC), miR-125a-5p mimics (miR-125a-5p),
miRNA inhibitors normal control (inh-NC) and miR-125a-5p inhibitors (miR-125a-5p-in) were provided by Guangzhou RiboBio Co., Ltd. The sequences were as follows: si-LIMK1, 5'-CTCCAGAGGGCTAAGTGTT-3';si-NC,5'-CTCGAGCGG AATTGCAGTT-3'; miR-125a-5p mimics, 5'-TCCCTGAGA CCCTTTAACCTGTGA-3'; miR-NC, 5'-UUCUCCGAACGU GUCACGUTT-3'; miR-125a-5p-in, 5'-TCACAGGTTAAAGG GTCTCAGGGA-3'; and inh-NC, 5'-ACGAUACACGUUCGG AGAATT-3'. When cells confluence was $\sim 50 \%$, Lipofectamine ${ }^{\circledR}$ 2000 (Invitrogen; Thermo Fisher Scientific Inc.) according to the manufacturer's instructions and all the aforementioned plasmids, siRNAs, inhibitors and mimics were transfected into CC cells. The transfection temperature was $37^{\circ} \mathrm{C}$ and lasted for $24 \mathrm{~h}$. The final concentration of oligonucleotides the was $50 \mathrm{nM}$ and labeled as miR-NC group, miR-125a-5p group, inh-NC group, miR-125a-5p-in group, NC group, LIMK1 group, si-NC group, si-LIMK1 group, LIMK1+miR-125a-5p group and si-LIMK1+miR-125a-5p-in group, respectively. After $24 \mathrm{~h}$, cells were analyzed to determine transfection efficiency using reverse transcription-quantitative (RT-q) PCR or used for subsequent experimentation.

RT-qPCR. After homogenization of the CC tissues or CC cells (C-33A, CaSKi, C-33A/DDP and CaSKi/DDP), TRIzol ${ }^{\circledR}$ (Invitrogen; Thermo Fisher Scientific Inc.) reagent was added to extract total RNA. Then, reverse transcription was performed using Takara reverse transcription kit (Takara Biotechnology Co., Ltd.). Temperature and duration for reverse transcription were $37^{\circ} \mathrm{C}$ for $15 \mathrm{~min}$ and $85^{\circ} \mathrm{C}$ for $5 \mathrm{~min}$. A PCR reaction system with a final volume of $20 \mu \mathrm{l}$ was established according to the manufacturer's instructions. A total of $2 \mu \mathrm{l}$ of cDNA, $10 \mu l$ of SYBR-Green qPCR Master Mix (Applied Biosystems), $0.5 \mu \mathrm{l}$ of upstream and downstream primers $(10 \mu \mathrm{mol} / \mathrm{l})$, and $\mathrm{ddH}_{2} \mathrm{O}$ was added to supplement the system volume to $20 \mu \mathrm{l}$. The thermocycling conditions were as follows: initial denaturation at $95^{\circ} \mathrm{C}$ for $5 \mathrm{~min} ; 45$ cycles of denaturation at $95^{\circ} \mathrm{C}$ for $15 \mathrm{sec}$, annealing at $60^{\circ} \mathrm{C}$ for $30 \mathrm{sec}$ and extension at $72^{\circ} \mathrm{C}$ for $20 \mathrm{sec}$. miR-125a-5p expression was normalized to U6, LIMK1 mRNA expression were normalized to GAPDH. The relative expression of mRNA was measured by $2_{-\Delta \Delta C q}$ method (15). The primers sequences used were presented in Table I.

MTT assay. C-33A/DDP cells and CaSKi/DDP cells were harvested and trypsinized. The cells were resuspended and inoculated into 96 -well plates at a density of $2 \times 10^{3}$ cells/well. On the 1st, 2nd and 3rd day of culture, $20 \mu \mathrm{l}$ of MTT solution $(5 \mathrm{mg} / \mathrm{ml})$ was added per well and incubated for $4 \mathrm{~h}$. Following that, the medium was removed and $150 \mu \mathrm{l}$ dimethyl sulfoxide (DMSO) was dripped into each well and oscillated by hand at room temperature for $10 \mathrm{~min}$. For the determination of the $\mathrm{IC}_{50}$ value of DDP, C-33A/DDP cells and CaSKi/DDP cells were seeded into 96-well plates and treated with a DDP concentration gradient of $0,2.5,5,10,20,40$ and $80 \mu \mathrm{g} / \mathrm{ml}$ for $24 \mathrm{~h}$. The absorbance (A) values at were recorded at $490 \mathrm{~nm}$ by a microplate reader. The cell proliferation rate and the inhibition rate of DDP were calculated using the following formulas: cell viability $(\%)=(\mathrm{A}$ value of control group- $\mathrm{A}$ value of specifically treated group)/A value of specifically treated group $\times 100 \%$; the inhibition rate of the drug to the cells $(\%)=(1-\mathrm{A}$ value of specifically treated group/A value of 
control group) $\mathrm{x} 100 \% . \mathrm{IC}_{50}$ was calculated by Probit analysis using the SPSS 22.0 software (IBM Corp.).

BrdU assay. Transfected C-33A/DDP and CaSKi/DDP cells were seeded at a density of 2,000 cells/well in a 96 well plate. Next, $48 \mathrm{~h}$ after transfection, cell proliferation was analyzed using the BrdU Cell Proliferation Assay kit (AmyJet Scientific Inc.) according to the manufacturer's instructions. All images were taken by a fluorescence microscope (magnification, x200). The counting of BrdU-positive cells was performed using ImageJ software v.1.52a (National Institutes of Health).

Flow cytometry analysis. Annexin V-FITC/propidium iodide (PI) Apoptosis Detection kit (cat. no. 40302ES20, Yeasen Biotech Co., Ltd.) was used to detect apoptosis. After being maintained for $72 \mathrm{~h}$, the transfected C-33A/DDP and $\mathrm{CaSKi} / \mathrm{DDP}$ cells were washed three times with PBS, then trypsinized and harvested from the medium. Subsequently, cells were resuspended with $500 \mu \mathrm{l}$ binding buffer, stained with Annexin V-FITC and PI and incubated for $20 \mathrm{~min}$ in the dark. Subsequently, apoptosis was detected by flow cytometry (Epics XL; Beckman Coulter Inc.) and FlowJo software v.10.4.2 (Treestar, Inc.) was used to analyze the data. Early and late apoptosis was assessed.

Western blotting. P-glycoprotein (P-gp) and Glutathione S-transferase- $\pi(\mathrm{GST}-\pi)$ are recognized as typical DDP chemoresistance protein markers $(16,17)$, hence their protein expression was assessed. Transfected C-33A/DDP and CaSKi/DDP cells were harvested and lysed with RIPA Lysis Buffer containing protease inhibitors (Thermo Fisher Scientific Inc.) for $30 \mathrm{~min}$. The supernatant was centrifuged at $4^{\circ}$ at the speed of $12,000 \mathrm{r} / \mathrm{min}(14,440 \mathrm{x} \mathrm{g})$ for $10 \mathrm{~min}$, and the protein concentration was determined using bicinchoninic acid (BCA) kit (Beyotime Institute of Biotechnology). Following that, $10 \mu \mathrm{g}$ protein of the supernatant was subjected to $12.5 \%$ SDS-PAGE and then transferred to PVDF membrane. Subsequently, the membrane was blocked with 5\% skimmed milk for $1 \mathrm{~h}$ at room temperature, incubated with primary antibody for $24 \mathrm{~h}$ at $4{ }^{\circ} \mathrm{C}$, then rinsed with TBST ( $0.1 \%$ Tween $)$ and incubated with secondary antibody (1:2,000; cat. no. ab205718) for another $1 \mathrm{~h}$ at room temperature. The primary antibodies used in this study including anti- $\beta$-actin antibody (1:500; cat. no. ab8227), anti-LIMK1 antibody (1:500; cat. no. ab81046,), anti-P-gp (1:500; cat. no. ab103477), and anti-GST- $\pi$ (1:500; cat. no. ab242014) were purchased from Abcam. Subsequently, the membrane was washed with TBST 3 times, and visualized using ECL (Guangzhou Xiang Bo Biological Technology Co., Ltd.). The intensity of each band was quantified using ImageJ software v.1.52a National Institutes of Health).

Bioinformatics analysis. The online software TargetScan (TargetScan Human 7.0; http://www.targetscan.org/vert_70/) was used for predicting downstream mRNAs of the miRNA.

Luciferase reporter gene assay. All luciferase reporter vectors [LIMK1-wild-type (WT) and LIMK1-mutant (MUT)] were constructed based on the pGL3 Luciferase Reporter Vector (Promega Corporation). C-33A/DDP and CaSKi/DDP cells were collected, and the reporter vectors were co-transfected
Table I. Primer sequences for RT-qPCR.

\begin{tabular}{lll}
\hline Name & \multicolumn{1}{c}{ Sequence (5'-3') } \\
\hline miR-125a-5p & F: CTGTCCCTGAGACCCTTTAAC \\
& R: CGAGGAAGAAGACGGAAGAAT \\
LIMK1 & F: CAAGGGACTGGTTATGGTGGC \\
& R: CCCCGTCACCGATAAAGGTC \\
U6 & F: CTCGCTTCGGCAGCACA \\
& R: AACGCTTCACGAATTTGCGT \\
GAPDH & F: AGAAGGCTGGGGCTCATTTG \\
& R: AGGGGCCATCCACAGTCTTC
\end{tabular}

miR, microRNA; LIMK1, LIM kinase 1; RT-q, reverse transcriptionquantitative; F, forward; R, reverse.

with miR-NC (5'-UUCUCCGAACGUGUCACGUTT-3') or miR-125a-5p mimics (5'-TCCCTGAGACCCTTTAACCTG TGA-3') with Lipofectamine ${ }^{\circledR} 2000$ (Invitrogen; Thermo Fisher Scientific Inc.) according to the manufacturer's instructions. After $24 \mathrm{~h}$, the treated cells were lysed with lysate and centrifuged at a speed of $14,440 \times \mathrm{g}$ for $10 \mathrm{~min}$ at $4^{\circ} \mathrm{C}$ and the supernatant was collected. Subsequently, the Dual-Luciferase Reporter Gene Assay kit (Promega Corporation) was used to measure luciferase activity according to the manufacturer's instructions. Relative luciferase activity was assayed $24 \mathrm{~h}$ after the transfection and normalized with Renilla luciferase activity.

Statistical analysis. All assays were repeated at least three times and the data were statistically analyzed using SPSS 22.0 (IBM Corp.). All data were expressed as mean \pm standard deviation (mean $\pm \mathrm{SD})$. Whether the data were normally distributed was examined by One-Sample Kolmogorov-Smirnov test. For normally distributed data, unpaired Student's t-test was used to make the comparison between 2 groups. one-way or two-way (as appropriate) ANOVA followed by the post hoc Tukey's test was used for multiple comparisons. $\mathrm{IC}_{50}$ of the cells to cisplatin was calculated by Probit analysis. Pearson's correlation analysis was used to analyze the correlation between miR-125a-5p expression and LIMK1 expression in CC samples. $\mathrm{P}<0.05$ was considered to indicate a statistically significant difference.

\section{Results}

miR-125a-5p is downregulated in CC tissues and cells compared with normal tissues and HUCECs. Firstly, RT-qPCR was used to detect the expression of miR-125a-5p in 45 pairs of $\mathrm{CC}$ cancer tissues and corresponding normal tissues, the findings of which indicated that miR-125a-5p expression in $\mathrm{CC}$ tissues was downregulated compared with normal tissues (Fig. 1A). Subsequently, C-33A/DDP and CaSKi/DDP cell lines were established and flow cytometry performed, the findings indicated that there were no tumor-suppressive effects of $10 \mu \mathrm{g} / \mathrm{ml}$ DDP on the apoptotic rate in C-33A/DDP and CaSKi/DDP cells (Fig. S1A). Western blotting demonstrated that compared with C-33A and CaSKi 
A

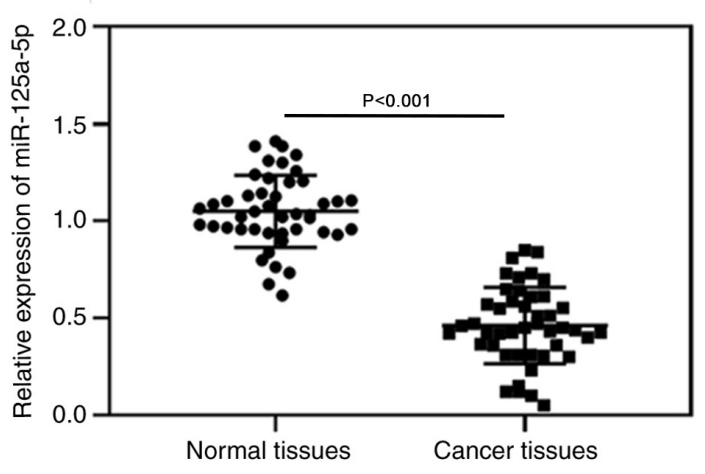

B

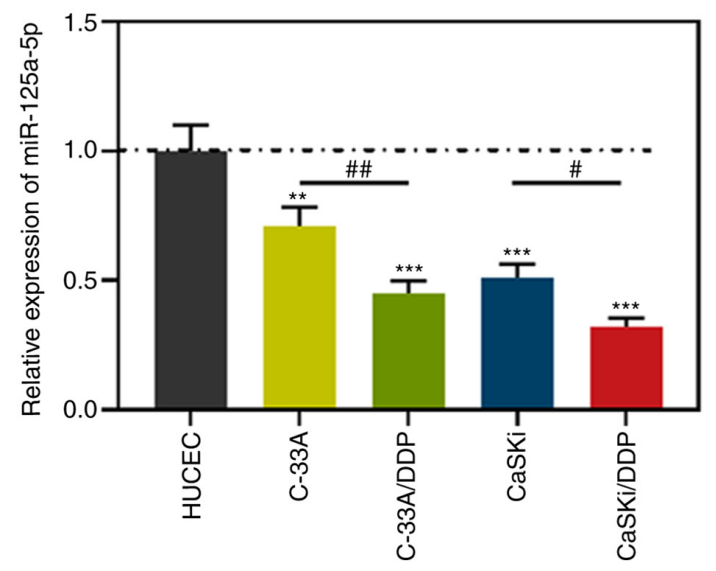

Figure 1. miR-125a-5p expression in CC tissues and cells. (A) RT-qPCR was used to detect miR-125a-5p expressions in normal and cancerous tissues of patients $(\mathrm{n}=45)$; (B) RT-qPCR was used to detect miR-125a-5p expressions in patients with HUCEC lines and cancer cell lines. ${ }^{* *} \mathrm{P}<0.01$ and ${ }^{* * *} \mathrm{P}<0.001$ vs. HUCEC; ${ }^{\#} \mathrm{P}<0.05$ and ${ }^{\# \#} \mathrm{P}<0.01$ vs. DDP-resistant cells. miR, microRNA; RT-q, reverse transcription-quantitative; CC, cervical carcinoma; DDP, cisplatin.

cells, protein expressions of P-gp and GST- $\pi$ in C-33A/DDP, and CaSKi/DDP cells were significantly upregulated (Fig. S1B). In addition, RT-qPCR was used to detect the expression of miR-125a-5p in normal cervical epithelial cells (HUCEC) and $\mathrm{CC}$ cell lines. The results demonstrated that compared with HUCEC cells, miR-125a-5p expression in CC cells was significantly downregulated, while the expression of miR-125a-5p in C-33A/DDP and CaSKi/DDP cells was significantly lower compared with C33A and CaSKi cells (Fig. 1B). These findings implied that the downregulation of miR-125a-5p was related to the occurrence of DDP resistance in CC cells.

miR-125a-5p reduces drug resistance of DDP-resistant $C C$ cells. RT-qPCR analysis demonstrated that, compared with miR-NC, the expression level of miR-125a-5p was significantly increased in CaSKi/DDP cells transfected with miR-125a-5p mimics; compared with inh-NC, while the expression level of miR-125a-5p was significantly decreased in C-33A/DDP cells transfected with miR-125a-5p inhibitors (Fig. 2A). Subsequent experiments revealed that compared with the miR-NC group, the cell viability and proliferation of the miR-125a-5p group were reduced, the apoptosis rate of the cells was increased, the $\mathrm{IC}_{50}$ value of DDP was reduced (Fig. 2B-G). Western blotting results demonstrated that $\mathrm{P}-\mathrm{gp}$ and GST- $\pi$ protein expression was downregulated in the miR-125a-5p mimic group. Conversely, compared with the inh-NC group, the cell viability and proliferation of the miR-125a-5p-in group were increased, the apoptosis rate of the cells was reduced, the $\mathrm{IC}_{50}$ value of DDP was increased, P-gp and GST- $\pi$ protein expression was upregulated (Fig. 2B-G). These results suggested that miR-125a-5p sensitized CC cells to DDP.

LIMK1 is a target gene of miR-125a-5p. By an online prediction with TargetScan, it was predicted that there was a potential binding site between LIMK1 and miR-125a-5p (Fig. 3A). In addition, the targeting relationship between miR-125a-5p and LIMK1 was validated by the luciferase reporter gene assay. It was found that the luciferase activity of LIMK1 WT cells transfected with miR-125a-5p mimics was lower compared with the miR-NC group. There were no significant changes observed in the luciferase activity of LIMK1 MUT cells (Fig. 3B). Pearson's correlation test was used to investigate the correlation between LIMK1 and miR-125a-5p and the results demonstrated that miR-125a-5p mRNA expression was negatively correlated with LIMK1 mRNA expression (Fig. 3C). Additionally, LIMK1 expression in the cells transfected with miR-125a-5p mimics was downregulated compared with the miR-NC group; meanwhile, the expression of LIMK1 in the cells transfected with miR-125a-5p inhibitors was upregulated compared with the inh-NC group (Fig. 3D-E). In summary, miR-125a-5p negatively regulated LIMK1 expression in CC cells.

LIMK1 is upregulated and enhances the drug resistance in DDP-resistant CC cells compared with normal tissues and HUCECs. LIMK1 mRNA expression was examined by RT-qPCR, the results indicated that compared with normal tissues, LIMK1 expression was enhanced in CC tissues (Fig. 4A). In addition, the expression of LIMK1 in CC cells was significantly upregulated compared with HUCEC cells, and LIMK1 expression was higher in C-33A/DDP and $\mathrm{CaSKi} / \mathrm{DDP}$ cells compared with $\mathrm{C}-33 \mathrm{~A}$ and $\mathrm{CaSKi}$ cells (Fig. 4B-C). Subsequently, C-33a/DDP cells with the lowest expression of LIMK1 were selected for overexpression experiments and CaSki/DDP cells with the highest expression of LIMK1 were chosen for knockdown experiments. RT-qPCR analysis also demonstrated that the expression level of LIMK1 significantly increased in C-33A/DDP cells transfected with LIMK1-overexpressing vector compared with NC group; while compared with si-NC group, the expression level of LIMK1 significantly decreased in CaSKi/DDP cells transfected with si-LIMK1 (Fig. 4D). Further experiments demonstrated that compared with the $\mathrm{NC}$ group, the proliferation activity of the CC cells in the LIMK1 overexpression group was increased, the apoptosis rate was reduced, the $\mathrm{IC}_{50}$ value of DDP was increased, and P-gp and GST- $\pi$ protein expressions were upregulated (Fig. 4E-I). In contrast, compared with the control group, the cell viability of the LIMK1 knockdown group was decreased, the apoptosis rate was enhanced, the $\mathrm{IC}_{50}$ value of DDP was decreased, and the protein expression level of 
A

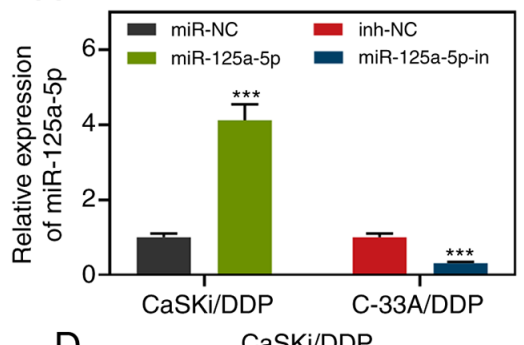

$\mathrm{D}$

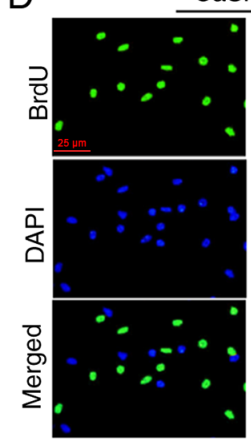

miR-NC

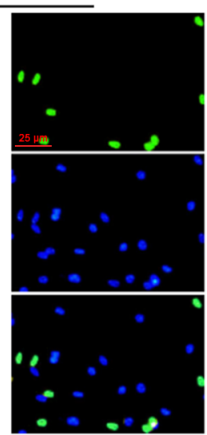

miR-125a-5p
B

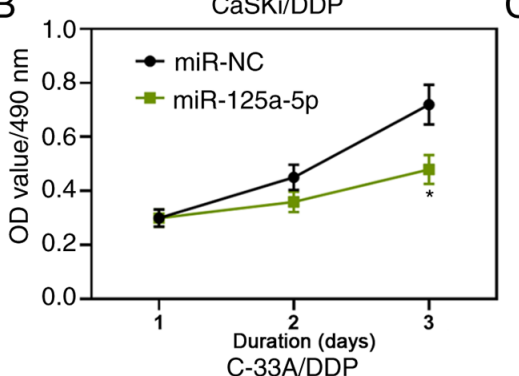

C
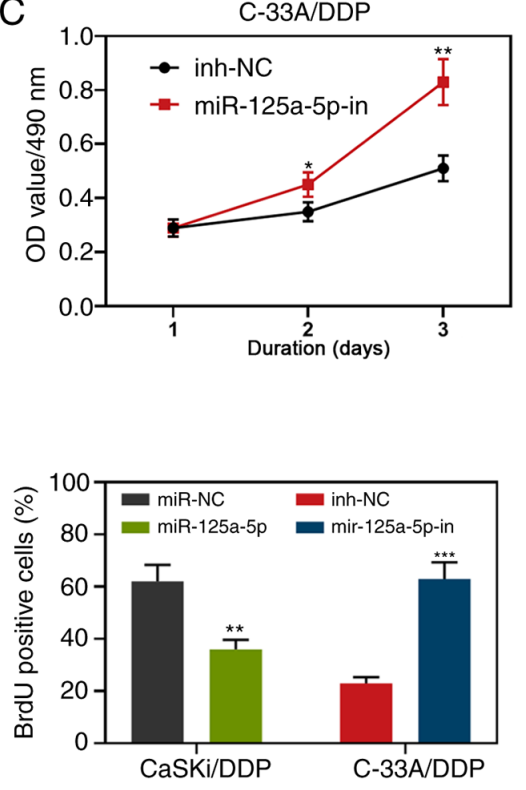
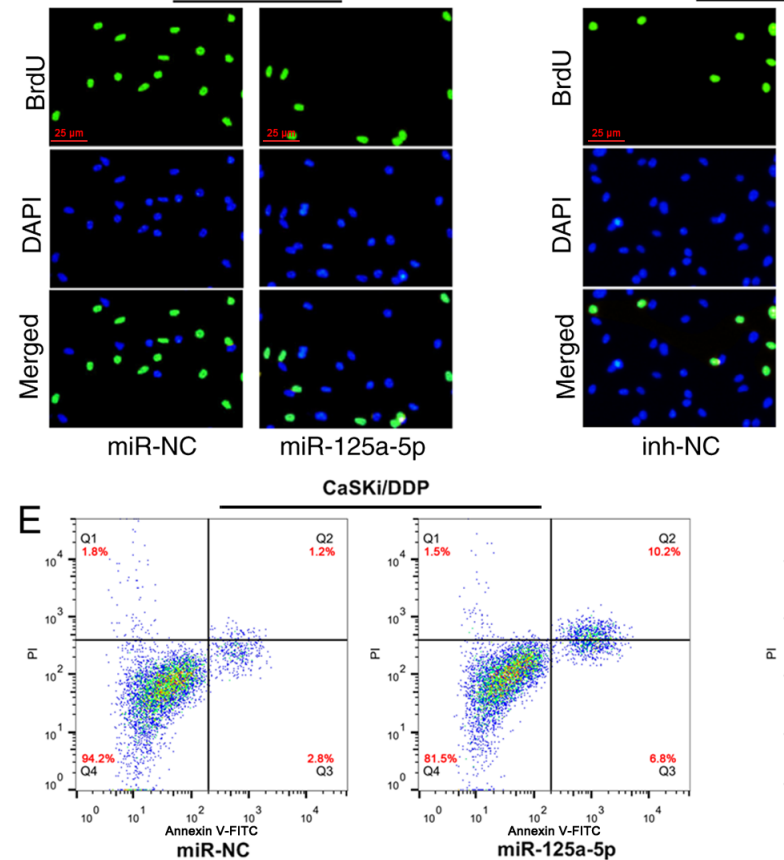

inh-NC

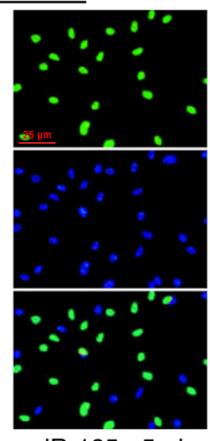

miR-125a-5p-in

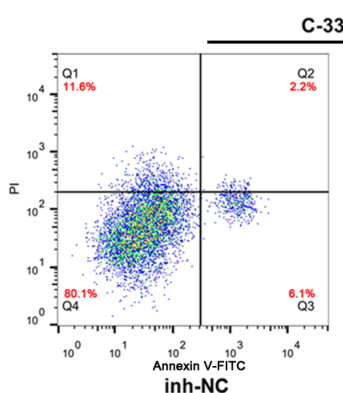

C-33A/DDP
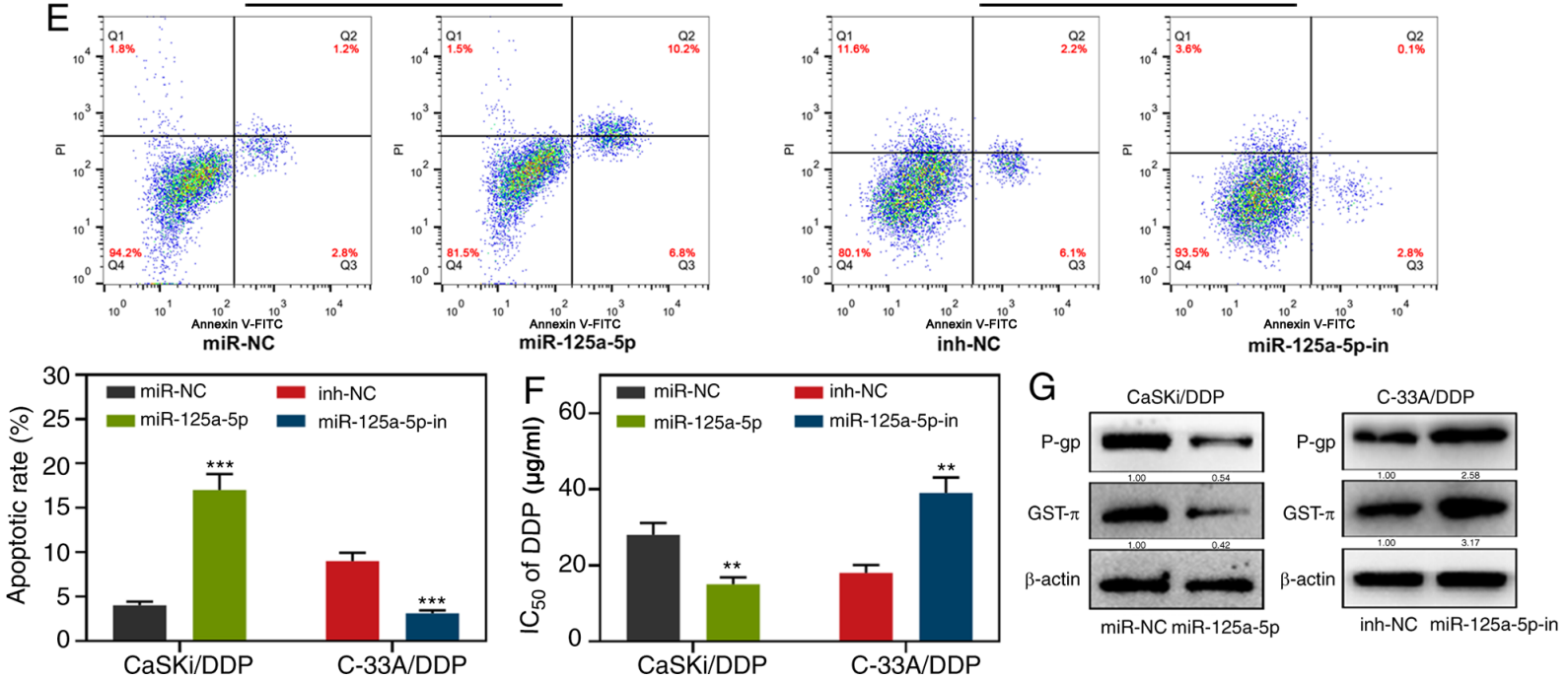

Figure 2. Effects of miR-125a-5p on CC cell proliferation, apoptosis, $\mathrm{IC}_{50}$ of DDP and chemoresistance-related proteins. (A) RT-qPCR was used to detect the transfection efficiency of miR-125a-5p mimics or miR-125a-5p inhibitors. (B-D) MTT assay and BrdU assay were used to detect the proliferation activity of CaSKi/DDP and C-33A/DDP cells after transfection with miR-125a-5p mimics or miR-125a-5p inhibitors. (E) Effects of miR-125a-5p mimics or miR-125a-5p inhibitors on apoptosis were detected by flow cytometry analysis. (F) MTT assay was used to detect the effect of miR-125a-5p mimics or miR-125a-5p inhibitors on $\mathrm{IC}_{50}$ of DDP. (G) Effects of miR-125a-5p mimics or miR-125a-5p inhibitors on the expression of apoptotic protein were detected by western blot. ${ }^{*} \mathrm{P}<0.05,{ }^{* *} \mathrm{P}<0.01$ and ${ }^{* * *} \mathrm{P}<0.001$. miR, microRNA; RT-q, reverse transcription-quantitative; CC, cervical carcinoma; DDP, cisplatin; NC, negative control; inh, inhibitor; OD, optical density; P-gp, anti-P-glycoprotein; GST- $\pi$, anti-glutathione S-transferase- $\pi$.

P-gp and GST- $\pi$ protein were decreased (Fig. 4E-I). These results indicated that LIMK1 enhanced the drug resistance of DDP-resistant CC cells and targeting LIMK1 reversed the chemoresistance.

miR-125a-5p reduces drug resistance of DDP-resistant $C C$ cells by absorbing LIMK1. To investigate the effect of miR-125a-5p targeting LIMK1 on the chemoresistance of CC cells, miR-125a-5p mimics and LIMK1 overexpressing plasmids, miR-125a-5p inhibitors and si-LIMK1 were co-transfected into DDP resistant cells. RT-qPCR analysis demonstrated that the transfections were successful (Fig. 5A). Further experiments revealed that compared with the NC group, the proliferation activity of cells in the LIMK1 group was enhanced, while miR-125a-5p mimics inhibited this effect (Fig. 5B-C). Also, LIMK1 inhibited the apoptosis rate of cells, while miR-125a-5p partially reversed this effect (Fig. 5D). Compared with the NC group, in the overexpressing LIMK1 group, the $\mathrm{IC}_{50}$ value of DDP was increased, and P-gp and GST- $\pi$ protein expression were upregulated, but miR-125a-5p could attenuate these effects (Fig. 5E-F). As expected, compared with the si-NC group, LIMK1 knockdown inhibited cell viability, promoted apoptosis, reduced the $\mathrm{IC}_{50}$ value of DDP to cells, and downregulated P-gp and GST- $\pi$ protein expression, whereas miR-125a-5p inhibitors negatively regulated these phenomena (Fig. 5B-F). These results implied 


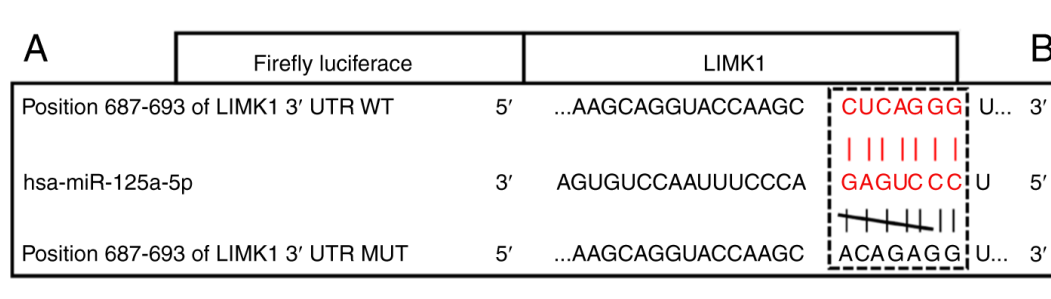

C

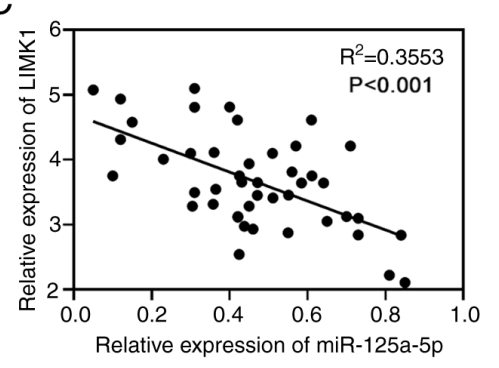

D

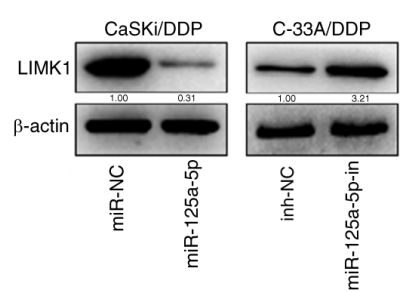

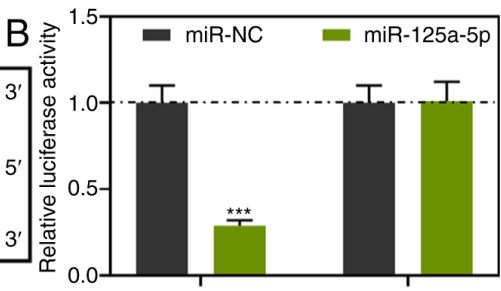

E

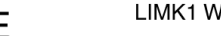

LIMK1 MUT

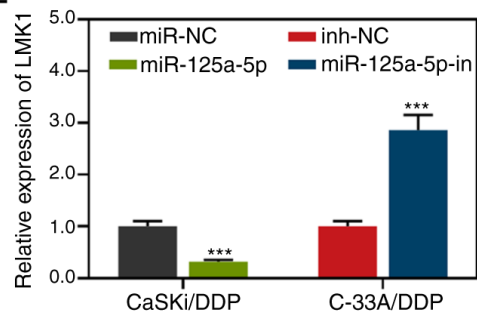

Figure 3. miR-125a-5p targets LIMK1. (A) Potential binding site between miR-125a-5p and LIMK1. (B) Luciferase reporter gene assay was used to validate the predicted binding site between miR-125a-5p and LIMK1. (C) Correlation between miR-125a-5p expression and LIMK1 expression in clinical CC samples. (D-E) Effects of miR-125a-5p on LIMK1 expressions were detected by western blotting. ${ }^{* * *} \mathrm{P}<0.001$ vs. miR-NC or inh-NC. miR, microRNA; CC, cervical carcinoma; DDP, cisplatin; NC, negative control; WT, wild-type; MUT, mutant; UTR, untranslated region; LIMK1, LIM kinase 1.

that the function of miR-125a-5p on modulating the chemoresistance of CC cells was dependent on LIMK1.

\section{Discussion}

Chemotherapy is a crucial therapy for patients with $\mathrm{CC}$ to prolong lifespan and improve quality of life, and DDP has been widely used in clinical CC treatments $(10,18)$. However, the resistance of cancer cells to DDP during treatments limits the efficacy of DDP (19). In recent years, numerous studies have demonstrated that miRNAs and mRNAs feature prominently in CC proliferation, metastasis, apoptosis and DDP resistance (20-22). The present study demonstrated that miR-125a-5p expression was downregulated in CC tissues and cell lines compared with normal tissues or HUCECs. The gain and loss of function experiments results indicated that miR-125a-5p targeted LIMK1 to inhibit CC cell proliferation, promote apoptosis and reduce drug resistance to DDP.

miR-125a-5p is differentially expressed in various cancers, such as colorectal cancer, bladder cancer, and breast cancer and serves as a tumor suppressor and can affect tumor cell proliferation, apoptosis and metastasis via regulating vascular endothelial growth factor A, fucosyltransferase 4, BRCA1 associated protein 1 and ABL proto-oncogene 2 (23-26). For instance, $\mathrm{miR}-125 \mathrm{a}-5 \mathrm{p}$ suppresses colorectal cancer progression by targeting VEGFA (23). In CC, miR-125a-5p downregulates ABL proto-oncogene 2 expression, thereby inhibiting cell proliferation and migration (26). With in-depth study, it has been found that miR-125a-5p can affect not only the growth and the metastasis of tumor cells, but also the drug resistance of tumor cells (27). In esophageal squamous cell carcinoma, miR-125a-5p expression is downregulated and the sensitivity of cancer cells to DDP is enhanced via activation of the STAT3 signaling pathway (28). In the present study, it was found that compared with normal tissues or HUCECs, the expression of miR-125a-5p was downregulated in CC tissues and cell lines; miR-125a-5p mimics could inhibit the expression of drug resistance-related proteins P-gp and GST- $\pi$, hinder cell proliferation, promote apoptosis and decrease $\mathrm{IC}_{50}$ value in DDP resistant cells. The results of transfection of miR-125a-5p inhibitors were opposite to the aforementioned results, indicating that miR-125a-5p could inhibit DDP resistance of CC cells.

LIMK1 serves an essential regulatory role in proliferation, metastasis and apoptosis of tumors, including colonic, ovarian, and gastric cancer (29-31). It has been reported that LIMK1 was highly expressed in NSCLC, and its high expression was closely associated with advanced TNM stage and lymph node metastasis; in addition, LIMK1 interference significantly increased the sensitivity of NSCLC cells to DDP, suggesting that LIMK1 has as a cancer-promoting role in NSCLC and enhances DDP resistance (14). In the present study, RT-qPCR and western blotting demonstrated that compared with normal tissues or HUCECs, the expression of LIMK1 was upregulated in CC tissues and cell lines. Overexpression of LIMK1 in the present study increased the proliferation, inhibited the apoptosis of DDP-resistant cells C-33A/DDP and CaSKi/DDP cells, increased the $\mathrm{IC}_{50}$, promoted the expression of resistance-related proteins $\mathrm{P}-\mathrm{gp}$ and GST- $\pi$ and enhance the DDP resistance in CC.

The miRNA-mRNA axis exerts a critical part in the progression of tumors, including CC (32-34). In recent years, numerous studies demonstrated that the miRNA-mRNA axis exerts a regulatory role in DDP resistance of CC, miR-130a was found to directly target copper transporter protein 1 resulting in upregulation of DDP resistance in CC (35). In the present study, the potential binding site between miR-125a-5p and LIMK1 was predicted using the TargetScan database, and the subsequent luciferase reporter activity assay demonstrated that miR-125a-5p significantly decreased luciferase activity of the LIMK1 WT reporter, but didn't reduce activity of the LIMK1 MUT reporter. Western blotting assay results demonstrated that miR-125a-5p negatively regulated the expression of LIMK1 protein. Additionally, by performing gain- and loss-of-function experiments, the present study demonstrated that miR-125a-5p impeded LIMK1 expression and reduced 
A

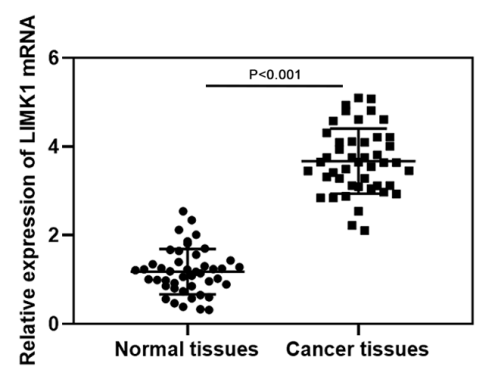

D

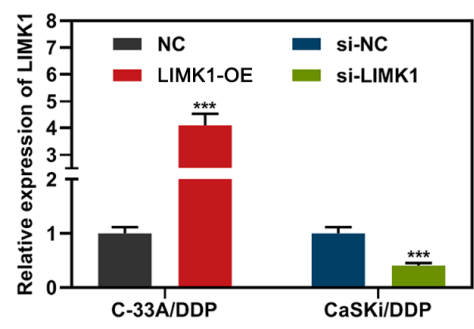

F

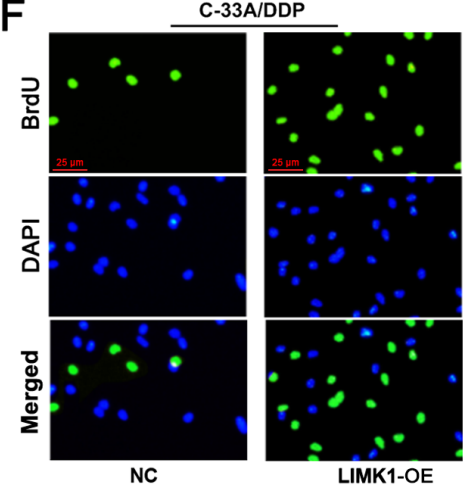

B

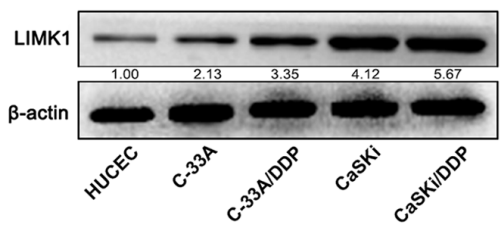

E

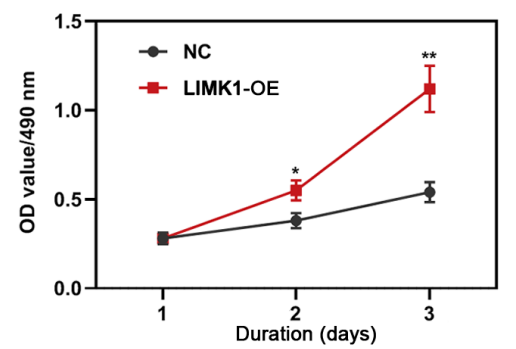

CaSKi/DDP

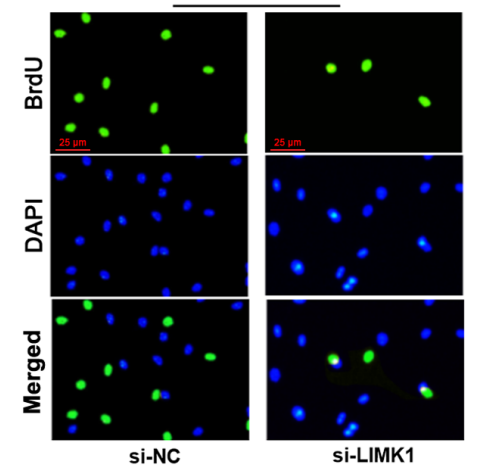

C

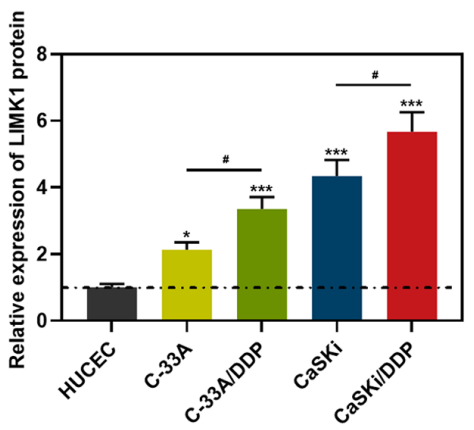

CaSKi/DDP
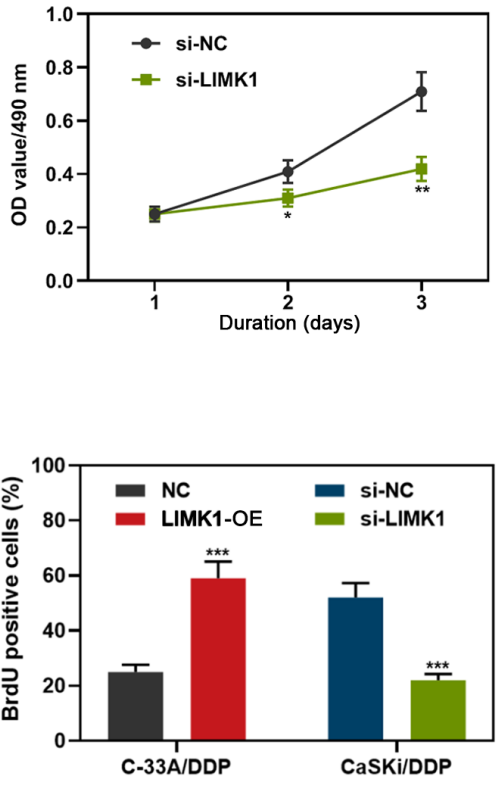

G

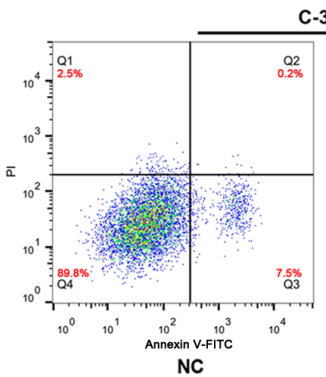

NC

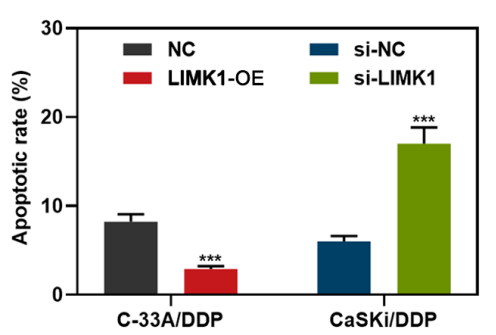

C-33A/DDP
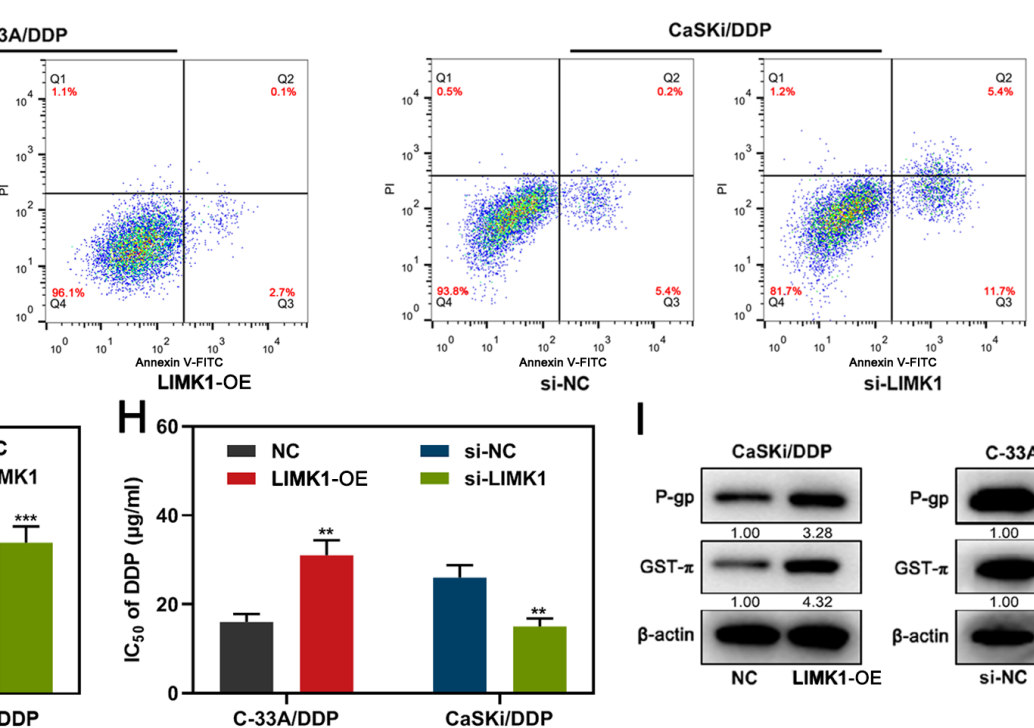

I
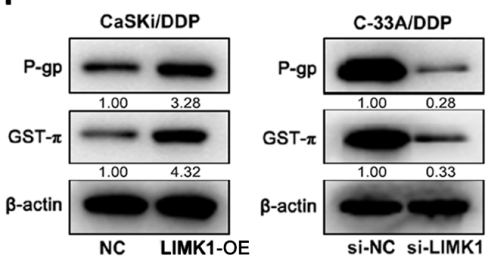

Figure 4. LIMK1 expression pattern in $\mathrm{CC}$ and the effects of it on proliferation, apoptosis, $\mathrm{IC}_{50}$ of DDP and drug resistance-related proteins in CC cells. (A-C) LIMK1 expression in CC tissues and cells were detected by RT-qPCR and western blotting. (D) RT-qPCR was used to detect the transfection efficiency of C-33A/DDP and CaSKi/DDP cells after transfection with LIMK1 overexpression plasmid or si-LIMK1. (E-F) MTT assay and BrdU assay were used to detect the proliferation activity of C-33A/DDP and CaSKi/DDP cells after transfection with LIMK1 overexpression plasmid or si-LIMK1. (G) Effects of LIMK1 overexpression plasmid or si-LIMK1 on apoptosis of CC cells. (H) MTT assay was used to detect the effect of LIMK1 overexpression plasmid or si-LIMK1 on $\mathrm{IC}_{50}$ of DDP. (I) Effects of LIMK1 overexpression plasmid or si-LIMK1 on the expression of chemoresistance-related proteins were detected by western blotting. ${ }^{*} \mathrm{P}<0.05,{ }^{* *} \mathrm{P}<0.01$ and ${ }^{* * *} \mathrm{P}<0.001$ vs. HUCEC, NC or si-NC. ${ }^{*} \mathrm{P}<0.05$ vs. DDP-resistant cells. miR, microRNA; RT-q, reverse transcription-quantitative; CC, cervical carcinoma; DDP, cisplatin; NC, negative control empty plasmid; OD, optical density; P-gp, anti-P-glycoprotein; GST- $\pi$, anti-glutathione S-transferase-ת; LIMK1, LIM kinase 1; si, small interfering; PI, propidium iodide; OE, overexpression. 
A
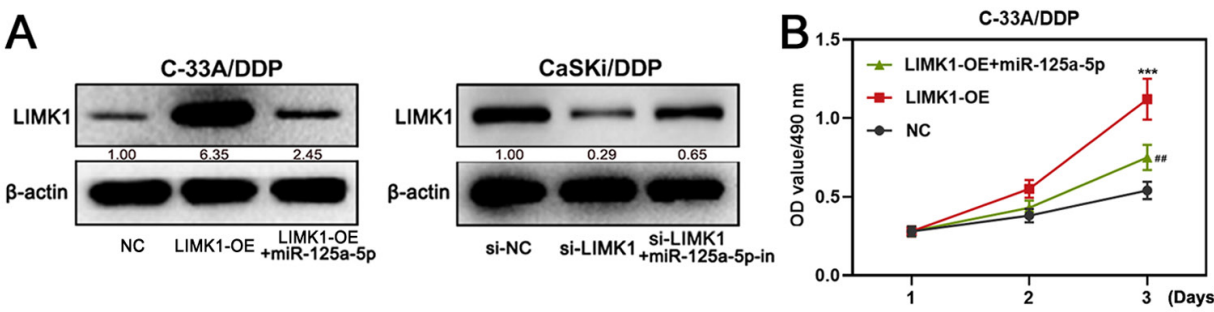

D

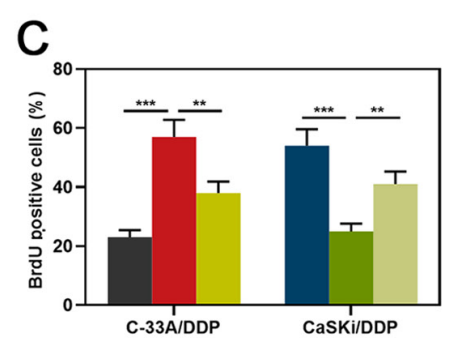

$\mathrm{E}$

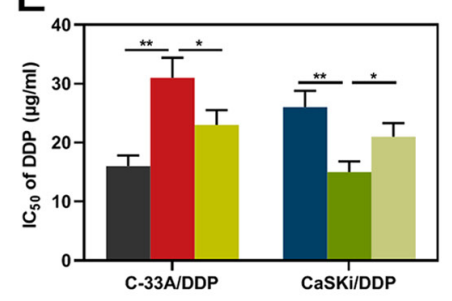

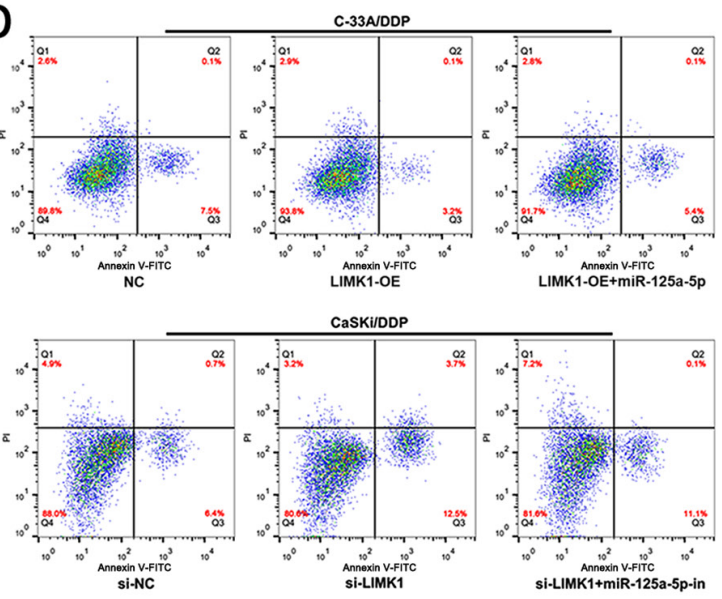

$\mathrm{F}$

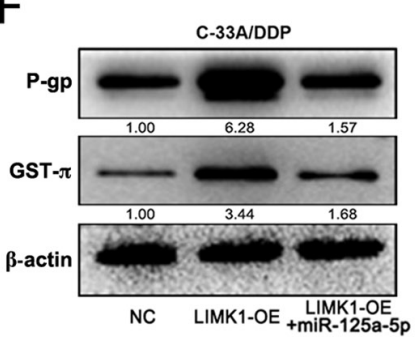

CaSKi/DDP
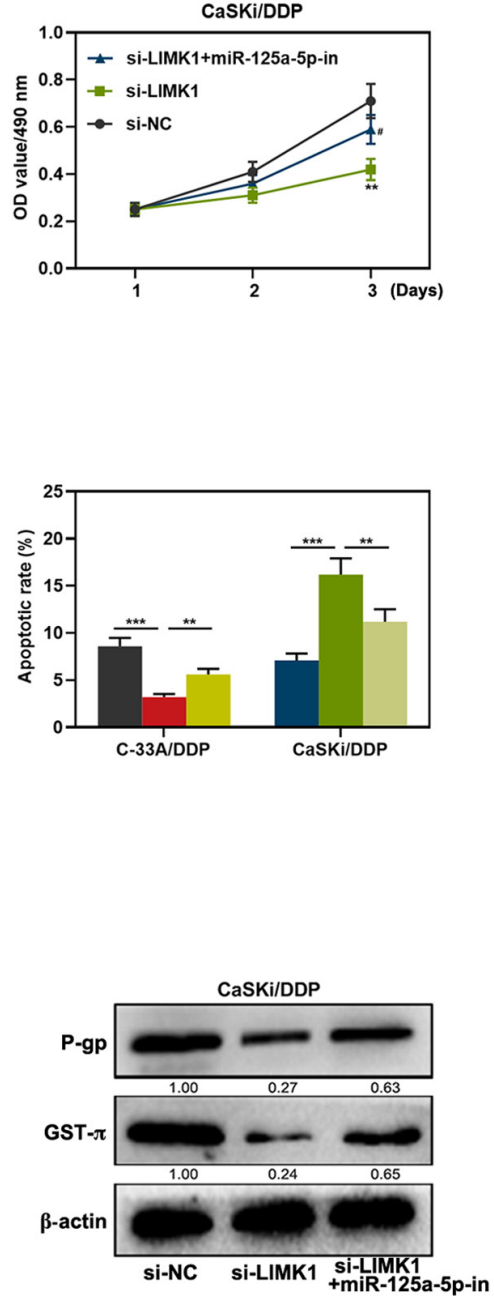

Figure 5. Effects of miR-125a-5p/LIMK1 axis on the proliferation, apoptosis, $\mathrm{IC}_{50}$ of DDP and drug resistance-related proteins in CC cells. (A) Expression of LIMK1 of C-33A/DDP and CaSKi/DDP cells after co-transfection with miR-125a-5p mimics and LIMK1 overexpression plasmid, miR-125a-5p inhibitors and si-LIMK1 were detected by western blotting. (B-C) MTT assay and BrdU assay were used to detect the proliferation activity of CC cells after transfection. (D) Flow cytometry was used to detect the apoptosis of CC cells. (E) MTT assay was used to detect the change of IC $_{50}$ of DDP after transfection. (F) Western blotting was used to detect the expression of chemoresistance-related proteins after transfection. ${ }^{*} \mathrm{P}<0.05,{ }^{* *} \mathrm{P}<0.01$, and ${ }^{* * * *} \mathrm{P}<0.001$ vs. NC or si-NC. ${ }^{\#} \mathrm{P}<0.05$, and ${ }^{\# \#} \mathrm{P}<0.01$ vs. LIMK1-OE or si-LIMK1. miR, microRNA; RT-q, reverse transcription-quantitative; CC, cervical carcinoma; DDP, cisplatin; NC, negative control empty plasmid; OD, optical density; P-gp, anti-P-glycoprotein; GST- $\pi$, anti-glutathione S-transferase- $\pi$; LIMK1, LIM kinase 1; si, small interfering; inh, inhibitor; PI, propidium iodide; OE, overexpression.

the resistance of CC cells to DDP. The findings of the present study not only partly explained the mechanism by which miR-125a-5p modulated the chemoresistance of CC cells, but also identified LIMK1 as a novel target gene of miR-125a-5p.

The present study had some limitations. Firstly, other target genes of miR-125a-5p were not identified. Future studies investigating this will help to further clarify the mechanism of miR-125a-5p in regulating the chemosensitivity of CC cells to DDP. Secondly, whether miR-125a-5p modulates the chemosensitivity of another chemotherapeutic drug apart from DDP deserves further investigation. Thirdly, the detailed mechanism by which miR-125a-5p/LIMK1 regulates the expression of P-gp and GST- $\pi$ is still unknown and should be the focus of future work.

In summary, compared with normal tissues or HUCECs, in CC tissue and cell lines, miR-125a-5p expression is downregulated and LIMK1 expression is upregulated. LIMK1 is a target gene of miR-125a-5p, which targets LIMK1 and inhibits the resistance to DDP of CC cells. The findings of the present study will improve the treatment of patients with CC.

\section{Acknowledgements}

Not applicable.

\section{Funding}

No funding was received.

\section{Availability of data and materials}

The datasets used and/or analyzed during the current study are available from the corresponding author on reasonable request.

\section{Authors' contributions}

PN, YX and YD conceived and designed the experiments. YX, YZ and LM performed the experiments. YX, YZ, YD and LM analyzed the results. YX, YZ, YD and PN wrote the paper. 
PN and YX confirm the authenticity of all the raw data. All authors have read and approved the final manuscript.

\section{Ethics approval and consent to participate}

The present study was given approval by the Medical Ethics Committee of Shengli Oilfield Central Hospital (Dongying, China; approval no, 2016-05). All patients provided written informed consent prior to surgery.

\section{Patient consent for publication}

Not applicable.

\section{Competing interests}

The authors declare that they have no competing interests.

\section{References}

1. Siegel RL, Miller KD and Jemal A: Cancer statistics, 2020. CA Cancer J Clin 70: 7-30, 2020.

2. Liu R, Qian M, Zhou T and Cui P: TP53 mediated miR-3647-5p prevents progression of cervical carcinoma by targeting AGR2 Cancer Med 8: 6095-6105, 2019.

3. Federico C, Sun J, Muz B, Alhallak K, Cosper PF, Muhammad N, Jeske A, Hinger A, Markovina S, Grigsby P, et al: Localized delivery of cisplatin to cervical cancer improves its therapeutic efficacy and ninimizes its side effect profile. Int J Radiat Oncol Biol Phys: Nov 27, 2020 (Epub ahead of print). doi: 10.1016/j. ijrobp.2020.11.052.

4. Matsuoka H, Murakami R, Abiko K, Yamaguchi K, Horie A, Hamanishi J, Baba T and Mandai M: UGT1A1 polymorphism has a prognostic effect in patients with stage IB or II uterine cervical cancer and one or no metastatic pelvic nodes receiving irinotecan chemotherapy: A retrospective study. BMC Cancer 20: 729, 2020

5. Zhu H, Luo H, Zhang W, Shen Z, Hu X and Zhu X: Molecular mechanisms of cisplatin resistance in cervical cancer. Drug Des Devel Ther 10: 1885-1895, 2016.

6. Zheng TL, Li DP, He ZF and Zhao S: miR-145 sensitizes esophageal squamous cell carcinoma to cisplatin through directly inhibiting PI3K/AKT signaling pathway. Cancer Cell Int 19: 250, 2019.

7. Mesci A, Huang X, Taeb S, Jahangiri S, Kim Y, Fokas E, Bruce J, Leong HS and Liu SK: Targeting of CCBE1 by miR-330-3p in human breast cancer promotes metastasis. Br J Cancer 116 . 1350-1357, 2017.

8. Qiu Z, Li H, Wang J and Sun C: miR-146a and miR-146b in the diagnosis and prognosis of papillary thyroid carcinoma. Oncol Rep 38: 2735-2740, 2017.

9. Okazaki J, Tanahashi T, Sato Y, Miyoshi J, Nakagawa T, Kimura T, Miyamoto H, Fujino Y, Nakamura F, Takehara M, et al: MicroRNA-296-5p promotes cell invasion and drug resistance by targeting Bcl2-related ovarian killer, leading to a poor prognosis in pancreatic cancer. Digestion 101: 794-806, 2020.

10. Feng Y,Zou W, Hu C, Li G, Zhou S, He Y, Ma F, Deng C and Sun L: Modulation of CASC2/miR-21/PTEN pathway sensitizes cervical cancer to cisplatin. Arch Biochem Biophys 623-624: 20-30, 2017.

11. Huang WK, Akçakaya P, Gangaev A, Lee L, Zeljic K, Hajeri P, Berglund E, Ghaderi M, Åhlén J, Bränström R, et al: miR-125a-5p regulation increases phosphorylation of FAK that contributes to imatinib resistance in gastrointestinal stromal tumors. Exp Cell Res 371: 287-296, 2018.

12. Shi B, Ma C, Liu G and Guo Y: miR-106a directly targets LIMK1 to inhibit proliferation and EMT of oral carcinoma cells. Cell Mol Biol Lett 24: 1, 2019.

13. Zhang H, Wang Y, Xing F, Wang J, Wang Y, Wang H, Yang Y and Gao Z: Overexpression of LIMK1 promotes migration ability of multidrug-resistant osteosarcoma cells. Oncol Res 19:501-509, 2011

14. Chen Q, Jiao D, Hu H, Song J, Yan J, Wu L and Xu LQ: Downregulation of LIMK1 level inhibits migration of lung cancer cells and enhances sensitivity to chemotherapy drugs. Oncol Res 20: 491-498, 2013.
15. Livak KJ and Schmittgen TD: Analysis of relative gene expression data using real-time quantitative PCR and the 2(-Delta Delta C(T)) method. Methods 25: 402-408, 2001.

16. Xue F, Xu Y, Song Y, Zhang W, Li R and Zhu X: The effects of sevoflurane on the progression and cisplatinum sensitivity of cervical cancer cells. Drug Des Devel Ther 13: 3919-3928, 2019.

17. Xiao Y, Liang MR, Liu CC, Wang YN, Zeng Y, Zhou J, Zhu HT, Wang Q, Zou Y and Zeng SY: Overexpression of P16 reversed the MDR1-mediated DDP resistance in the cervical adenocarcinoma by activating the ERK1/2 signaling pathway. Cell Div 14: 6, 2019.

18. Conte E, Bresciani E, Rizzi L, Cappellari O, De Luca A, Torsello A and Liantonio A: Cisplatin-Induced skeletal muscle dysfunction: Mechanisms and counteracting therapeutic strategies. Int J Mol Sci 21: E1242, 2020.

19. Yi SA, Kim GW, Yoo J, Han JW, Kwon SH. HP1 $\gamma$ sensitizes cervical cancer cells to cisplatin through the suppression of UBE2L3. Int J Mol Sci 21: 5976, 2020.

20. Xu X, Jiang X, Chen L, Zhao Y, Huang Z, Zhou H and Shi M: miR-181a promotes apoptosis and reduces cisplatin resistance by inhibiting osteopontin in cervical cancer cells. Cancer Biother Radiopharm 34: 559-565, 2019.

21. Huang LL and Rao W: SiRNA interfering STAT3 enhances DDP sensitivity in cervical cancer cells. Eur Rev Med Pharmacol Sci 22: 4098-4106, 2018.

22. Xia J, Yu X, Song X, Li G, Mao X and Zhang Y: Inhibiting the cytoplasmic location of HMGB1 reverses cisplatin resistance in human cervical cancer cells. Mol Med Rep 15: 488-494, 2017.

23. Yang X, Qiu J, Kang H, Wang Y and Qian J: miR-125a-5p suppresses colorectal cancer progression by targeting VEGFA. Cancer Manag Res 10: 5839-5853, 2018.

24. Zhang Y, Zhang D, Lv J, Wang S and Zhang Q: miR-125a-5p suppresses bladder cancer progression through targeting FUT4. Biomed Pharmacother 108: 1039-1047, 2018.

25. Yan L, Yu MC, Gao GL, Liang HW, Zhou XY, Zhu ZT, Zhang CY, Wang YB and Chen X: miR-125a-5p functions as a tumour suppressor in breast cancer by downregulating BAP1. J Cell Biochem 119: 8773-8783, 2018.

26. Qin X, Wan Y, Wang S and Xue M: MicroRNA-125a-5p modulates human cervical carcinoma proliferation and migration by targeting ABL2. Drug Des Devel Ther 10: 71-79, 2015.

27. Liu R, Wang M, Li E, Yang Y, Li J, Chen S, Shen WJ, Azhar S, Guo Z and Hu Z: Dysregulation of microRNA-125a contributes to obesity-associated insulin resistance and dysregulates lipid metabolism in mice. Biochim Biophys Acta Mol Cell Biol Lipids 1865: 158640, 2020.

28. Zhao Y, Ma K, Yang S, Zhang X, Wang F, Zhang X, Liu H and Fan Q: MicroRNA-125a-5p enhances the sensitivity of esophageal squamous cell carcinoma cells to cisplatin by suppressing the activation of the STAT3 signaling pathway. Int J Oncol 53: 644-658, 2018

29. Su J, Zhou Y, Pan Z, Shi L, Yang J, Liao A, Liao Q and Su Q: Downregulation of LIMK1-ADF/cofilin by DADS inhibits the migration and invasion of colon cancer. Sci Rep 7: 45624, 2017.

30. Chen P, Zeng M, Zhao Y and Fang X: Upregulation of Limk1 caused by microRNA-138 loss aggravates the metastasis of ovarian cancer by activation of Limk1/cofilin signaling. Oncol Rep 32: 2070-2076, 2014.

31. You T, Gao W, Wei J, Jin X, Zhao Z, Wang C and Li Y: Overexpression of LIMK1 promotes tumor growth and metastasis in gastric cancer. Biomed Pharmacother 69: 96-101, 2015.

32. Wei WF, Zhou CF, Wu XG, He LN, Wu LF, Chen XJ, Yan RM, Zhong M, Yu YH, Liang L, et al: MicroRNA-221-3p, a TWIST2 target, promotes cervical cancer metastasis by directly targeting THBS2. Cell Death Dis 8: 3220, 2017.

33. Peng Y,Zhang X, Ma Q, Yan R, Qin Y, Zhao Y, Cheng Y, Yang M, Wang Q, Feng X, et al: MiRNA-194 activates the Wnt/ $\beta$-catenin signaling pathway in gastric cancer by targeting the negative Wnt regulator, SUFU. Cancer Lett 385: 117-127, 2017.

34. Hu S, Zheng Q, Wu H, Wang C, Liu T and Zhou W: miR-532 promoted gastric cancer migration and invasion by targeting NKD1. Life Sci 177: 15-19, 2017.

35. Feng C, Ma F, Hu C, Ma JA, Wang J, Zhang Y, Wu F, Hou T, Jiang S, Wang Y, et al: SOX9/miR-130a/CTR1 axis modulates DDP-resistance of cervical cancer cell. Cell Cycle 17: 448-458, 2018.

This work is licensed under a Creative Commons Attribution-NonCommercial-NoDerivatives 4.0 International (CC BY-NC-ND 4.0) License. 